\title{
O uso das redes sociais para o fortalecimento dos movimentos culturais
}

\author{
The use of social networks for the strengthening of cultural movements
}

El uso de las redes sociales para el fortalecimiento de los movimientos culturales

Gleycivânia Pacheon Borges ${ }^{1 *}$, Luana Frigulha Guisso².

\begin{abstract}
RESUMO
Objetivo: Este trabalho tem como objetivo contribuir para o fortalecimento dos movimentos culturais mediante ao uso das redes sociais e suas potencialidades. Métodos: Por se tratar de uma revisão de literatura, para este trabalho se fez necessário um levantamento de obras bibliográficas que tratam da cultura e da comunicação digital, a fim de compreender os mesmos. Revisão Bibliográfica: A cultura apresenta-se como um dos principais segmentos de uma cidade ou região, sendo importante a sua valorização e disseminação para toda a comunidade. No cenário atual, emerge a necessidade de se utilizar espaços que contribuam para o fortalecimento desse setor, para isto as redes sociais podem ser um forte aliado na promoção da cultura, contribuindo para o compartilhamento e interação dos indivíduos. Conclusão: Conclui-se que as redes sociais são espaços pertinentes para a interação e o fortalecimento dos movimentos culturais, apresentando-se como uma forma barata e rápida de proliferação das informações.
\end{abstract}

Palavras-chave: Movimentos culturais, Fortalecimento, Redes sociais.

\begin{abstract}
Objective: This work aims to contribute to the strengthening of cultural movements through the use of social networks and their potentialities. Methods: Because this is a literature review, this work required a survey of bibliographical works dealing with culture and digital communication in order to understand them. Bibliographic Review: The culture presents itself as one of the main segments of a city or region, being important its valorization and dissemination for the whole community. In the current scenario, there is a need to use spaces that contribute to the strengthening of this sector, for this social networks can be a strong ally in the promotion of culture, contributing to the sharing and interaction of individuals. Conclusion: It is concluded that social networks are relevant spaces for the interaction and strengthening of cultural movements, presenting itself as a cheap and rapid form of information proliferation.
\end{abstract}

Keywords: Cultural movements, Strengthening, Social networks.

\begin{abstract}
RESUMEN
Objetivo: Este trabajo tiene como objetivo contribuir al fortalecimiento de los movimientos culturales mediante el uso de las redes sociales y sus potencialidades. Métodos: Por tratarse de una revisión de literatura, para este trabajo se hizo necesario un levantamiento de obras bibliográficas que tratan de la cultura y de la comunicación digital, a fin de comprender los mismos. Revisión Bibliográfica: La cultura se presenta como uno de los principales segmentos de una ciudad o región, siendo importante su valorización y diseminación para toda la comunidad. En el escenario actual, surge la necesidad de utilizar espacios que contribuyan al fortalecimiento de ese sector, para esto las redes sociales pueden ser un fuerte aliado en la promoción de la cultura, contribuyendo al compartir e interacción de los individuos. Conclusión: Se concluye que las redes sociales son espacios pertinentes para la interacción y el fortalecimiento de los movimientos culturales, presentándose como una forma barata y rápida de proliferación de las informaciones.
\end{abstract}

Palabras clave: Movimientos culturales, Fortalecimiento, Redes sociales.

${ }^{1}$ Publicitária, Professora da Faculdade Vale do Cricaré. São Mateus-ES

2 Doutoranda em História Social das Relações Políticas pela UFES - Universidade Federal do Espírito Santo. São Mateus ES. *E-mail: gleycii.mm@hotmail.com

SUBMETIDO EM: 11/2018

ACEITO EM: $12 / 2018$

PUBLICADO EM: 4/2019

REAS/EJCH | Vol. Sup. 22 | e587 | DOI: https://doi.org/10.25248/reas.e587.2019 Página 1 de 6 


\section{INTRODUÇÃO}

As mídias e as redes sociais têm se mostrado importantes ferramentas de comunicação e publicidade empresarial. As redes sociais permitem rápida e fácil divulgação dos mais variados assuntos, além do acesso facilitado e a troca de interações que os torna uma grande comunidade virtual. Com esse grande volume de usuários e de informações disponíveis o uso dessas ferramentas torna-se essencial na vida diária das pessoas, podendo ser uma grande aliada para o fortalecimento e divulgação dos movimentos socioculturais (GABRIEL M, 2010).

Segundo Aguiar (2007) as redes sociais, constituem-se como importantes espaços que permitem ampliar as potencialidades dos indivíduos de se agruparem e agirem coletivamente diante de transformações ocorridas na tecnologia, cultura e sociedade. Com o desenvolvimento tecnológico e o crescimento das organizações da Internet, a web tem se fortalecido cada vez mais (COSTA AC, 2015).

O Objetivo Geral deste trabalho é de contribuir para o fortalecimento de movimentos culturais através dos usos das novas tecnologias, no caso deste trabalho, as redes sociais. Para isto se fez necessário identificar as técnicas de comunicação digital e o crescimento das redes sociais, avaliando a utilização destas redes para o fortalecimento de grupos culturais a análise que se pretende realizar com este artigo está voltada para as redes sociais, em especial pelas que propiciam o desenvolvimento de ações coletivas dos movimentos sociais e culturais. Alguns destes têm desenvolvido capacidades de articulação em redes virtuais, visando o aumento do compartilhamento de conhecimentos e experiências, bem como o aumento da amplitude da mobilização, da influência e da interlocução em relação às amplitudes de poder.

\section{COMUNICAÇÃO DIGITAL E AS REDES SOCIAIS}

Desde a década de 1990 com a origem da Internet, a conexão entre as pessoas tornou-se mais fácil. O conceito de Rede Social refere-se ao comportamento da sociedade, sendo um amplo espaço de democratização e compartilhamento de milhares de informações. Por sua vez provocou uma verdadeira revolução e tornou-se o espaço que permite pessoas e organizações a estabelecerem diversas relações que vão desde a partilha de informações a realização de atividades comerciais, interligando ao sistema digital um ambiente estruturado para a conexão social entre pessoas e encurtando distâncias (COSTA AC, 2015).

As autoras Ivone Laurdo Oliveira e Marlene Marchiori apresentam aspectos da revolução tecnológica e comunicação que aconteceu no mundo, conforme as autoras o progresso da tecnologia digital trouxe modificações que modificaram todos os campos da vida humana e suas atividades, além de ter diminuído a distância entre o ser humano e aquilo que ele propõe alcançar (OLIVEIRA IL e MARCHIORI M, 1999).

Essa comunicação se tornou essencial para a compreensão do tempo e para a sobrevivência dos indivíduos em sociedade nos dias atuais. Essa conectividade entre o ser humano nos remete aos primórdios dessa evolução, onde o homem passou a se comunicar primeiro por meio da associação dos elementos presentes em seu ambiente social e cultural. Essa rede social começou antes de qualquer estabelecimento de tecnologias transformadoras. O conceito está ligado ao que se pode denominar de diálogo, é a popularização do uso de ferramentas móveis que tem aumentado o poder de comunicação da população resultando em maior audiência na internet. As redes sociais costumam reunir uma motivação comum, porém podem se manifestar de diferentes formas num espaço coletivo e de projeção de ideais.

Na verdade, essas redes sempre existiram e, com o passar do tempo, foram crescendo e se expandindo. Segundo Savazone a era da informação está cada vezes mais organizada em redes, essas redes constituem uma nova organização na sociedade, onde as experiencias, o saber e a cultura se modificam de forma substancial. (SAVAZONE R e COHN S, 2009) Essas redes de informação foram essenciais para aquisição, construção e produção de conhecimento. Hoje milhões de pessoas podem se conectar no mesmo instante, em tempo real. Do Brasil ao Japão, todos conectados. O conceito de rede tem sido utilizado na definição de novas formas de socialização e do fluxo de informações dentro de uma ordem global. Mas essa é uma das diversas análises feitas sobre esse assunto.

REAS/EJCH | Vol. Sup. 22 | e587 | DOI: https://doi.org/10.25248/reas.e587.2019 Página 2 de 6 
O filósofo tunisiano-francês Pierre Lévy (2007) nos traz o conceito da "tecnologias da inteligência", onde, com a informática mudam-se todos os paradigmas preestabelecidos. Fotos que podem ser alteradas, costumes são mudados, leis, ciência, a informática potencializa inteligências e permite que seja construída uma ordem mais justa, mais igualitária para a humanidade.

Rede social é um ambiente onde pessoas com interesses em comum interagem entre si em busca de trocas de informações sobre um determinado tema. Ela tem como característica, por sua porosidade e abertura, a possibilidade de proporcionar uma relação horizontal e não hierárquica, como se pode perceber em outros tipos de redes. Essas redes permitem ultrapassar barreiras de tempo e espaço, podendo teoricamente abranger um número ilimitado de pessoas, são referência quando falamos em coletividade e interação, seu conceito está ligado ao que se pode denominar de diálogo (GABRIEL M, 2010).

O autor Pierre Lévy (1999) aborda o Cibespaço, como um suporte da inteligência coletiva, uma das principais condições de seu próprio desenvolvimento. Toda a história da cibercultura testemunha largamente sobre esse processo de retroação positiva, ou seja, sobre a automanutenção da revolução das redes digitais. Desse modo, apresenta fortes indícios do crescimento do ciberespaço e as formas interativas por meio das redes sociais. Em primeiro lugar, o crescimento do ciberespaço não determina automaticamente 0 desenvolvimento da inteligência coletiva, apenas fornece a esta inteligência um ambiente propício.

Pierre Lévy (1999) apresenta a trajetória do surgimento das Redes Sociais no mundo, surgiu em 1995 nos Estados Unidos e Canada a Classmates, que foi a primeira Rede Social com o objetivo de conectar estudantes da faculdade. A partir de então, as redes sociais se popularizaram até os dias de hoje, com o surgimento das redes de música como o programa Last.FM, fotos como o uso do programa flickr e vídeo com a rede social Vimeo. A Web, mais especificamente as redes sociais, configura um novo cenário para interação social, mesmo que virtual.

Os indivíduos criaram vínculos e relacionamentos, e possuem toda uma vida estruturada no cyberespaço transformando as formas de interação entre eles mesmos e como se relacionam com as empresas. A comunicação agora tem mais conectividade e mobilidade, as pessoas e as empresas agora têm um canal, uma ligação entre si para maior facilidade de interação. O relacionamento com uma marca pode, por exemplo, começar em uma curtida em uma foto ou a pessoa seguir o perfil da marca e assim passar a receber notícias sobre ela (GABRIEL M, 2010).

Em uma pesquisa realizada pela empresa ComScore podemos perceber o aumento dos usuários de internet no Brasil, em 2014, 90,8\% dos usuários de internet acessaram redes sociais e passaram aproximadamente $18 \%$ do seu tempo online navegando em sites. O público jovem é um dos grandes responsáveis por referendar esses dados, já que são a grande maioria neste meio. (COMSCORE, 2014)

Segundo informações da empresa comScore, o Brasil representa $40 \%$ da audiência de internet na américa latina e mais de $80 \%$ dos brasileiros estão conectados as redes sociais, tornando este espaço cada vez mais forte e interativo. Em um levantamento da Kantar IBOPE 83\% dos usuários de internet navegaram em suas redes sociais nos últimos 30 dias, mostrando que acessar redes sociais é uma das atividades preferidas dos internautas brasileiros. Em sequência está a atividade assistir e/ou baixar vídeos (69\%), ler notícias nacionais e internacionais (61\%) e ouvir música (38\%) (COMSCORE, 2014).

Ainda dentro desta mesma pesquisa, foi relatado que $44 \%$ os internautas concordam com a afirmação de que a "internet é a minha principal fonte de entretenimento", sendo que $59 \%$ buscam qualquer tipo de informação primeiro na internet. A pesquisa representa os hábitos de $47 \%$ da população brasileira entre $12 \mathrm{e}$ 75 anos, o equivalente a 83 milhões de pessoas.

Segundo o Relatório Digital in 2017, do site We Are Social, cerca de 19 milhões de novos usuários se conectaram as redes sociais mais usadas no Brasil, entre elas, o Instagram e o Facebook. Este número representa quase $10 \%$ da população do país. Além desse número, outro ponto interessante é a finalidade do 
uso das mídias sociais, $67 \%$ afirmam usá-las a fim de entretenimento, mas também buscam informações e notícias por meio de alguma mídia digital.

É notório o crescimento de participação dos usuários dessas redes no Brasil, alguns fatores macro ambientais vêm contribuindo de forma decisiva para o crescimento significativo das redes sociais e do próprio uso da internet no cenário nacional; A popularização e acessibilidade econômica aos aparelhos denominados smartphones e tablets; A disponibilidade do sistema wireless (rede sem fio) em ambientes públicos e privados; A interação do público brasileiro, em especial os jovens, a essas redes virtuais (COSTA, 2015).

Através das plataformas digitais é possível a utilização da comunicação de forma nunca vista antes. As informações navegam mais rapidamente, atingindo pessoas como consumidores e possíveis produtores, disseminando informação e fazendo desta um novo meio de marketing, menos invasivo, porém mais eficaz e de custo reduzido (KOTLER K, 2017).

\section{IMPACTOS, REDES SOCIAIS E PROCESSO CULTURAL}

O processo de globalização possibilita, de forma direta ou indireta, influência sobre os aspectos da existência, na vida econômica, cultural, nas relações interpessoais e na própria subjetividade. Um exemplo é a cultura. Os movimentos culturais fazem parte da essência de uma sociedade, a cultura pode ser considerada um dos principais elementos para a formação de um indivíduo. Com o advento da internet e o surgimento das redes sociais, os usuários de todas as partes do mundo começaram a trocar experiências e vivências, com essas trocas as informações e as opiniões foram ganhando cada vez mais força em diversos setores (SAVAZONE R e COHN S, 2009).

A palavra cultura deriva do latim, colere, significa "cultivar", partindo dessa ideia, pode-se perceber que a cultura é uma herança herdada por cada povo, nação e comunidade, que carrega consigo valores singulares. Os movimentos culturais fazem parte da essência de uma sociedade, a cultura pode ser considerada um dos principais elementos para a formação de um indivíduo (CHAUí M, 1986).

Outro exemplo de interpretação da relação entre cultura e civilização é dado por (Voltaire e Kant), segundo os quais a cultura seria um impulso natural humano para evoluir e aperfeiçoar sua moral e consciência, tornando-se assim medida de uma Civilização, meio para avaliar seu grau de desenvolvimento e progresso (CHAUI M, 1986).

A cultura pode ser associada a costumes, valores e vivências de cada indivíduo, estes são hábitos e aptidões adquiridos pelo ser humano não somente na família, mas também em todos os setores da sociedade da qual é membro. Pelas ciências sociais a cultura pode ser explicada como um conjunto de ideias, comportamentos, símbolos e práticas sociais, aprendidos de geração em geração através da vida em sociedade. No âmbito popular, a cultura pode ser representada pela raiz e criação de um povo, sua literatura, música, arte e dança. Esta, é influenciada pelas crenças de um povo e formada através da interação dos indivíduos. Esse sujeito, é formado na relação com "outras pessoas importantes para ele", que mediavam valores, os sentidos, símbolos e a cultura dos mundos que ele habita (CHAUI M, 1986).

Entretanto, os elementos culturais de uma comunidade ou de um indivíduo não se mantêm unificado e estável, pois a cultura é um processo, composto por transformações a partir das relações ou dos sistemas culturais que nos rodeiam (CHAUI M, 1986).

Para a cultura, essa disseminação de saberes, trouxe um mundo de possibilidades para o crescimento e fortalecimento de variadas crenças, instituições e movimentos. A Internet tornou-se um importante laboratório social para experimentação com as construções de identidade que caracterizam a vida pós-moderna. (OLIVEIRA IL e MARCHIORI M, 1999). 
A Internet teve uma taxa de penetração mais rápida do que qualquer outro meio na história. Nos Estados Unidos, a rádio levou trinta anos para chegar a sessenta milhões de pessoas, A TV atingiu esse nível de difusão em 15 anos, a Internet o fez em apenas três anos após a criação da web mundial. (BURKE, 2006)

A Internet incentiva a criação de novas comunidades, comunidades virtuais, Howard Rheingold em seu livro pioneiro comunidades virtuais, enfatiza o nascimento de uma nova forma de comunidade, que traz pessoas on-line em torno de valores e interesses, ele propôs a ideia de que comunidades online poderiam ser transformadas em reuniões físicas, festas amigáveis e suporte material para membros da comunidade virtual. Em geral, entende-se que a comunidade virtual, de acordo com o argumento de Rheingold, é uma rede eletrônica autodefinida de comunicações interativas e organizada em torno de interesses ou fins em comum, embora às vezes a comunicação se torne o objetivo em si. (GABRIEL M, 2010)

Culturas consistem em processos de comunicação. E todas as formas de comunicação são baseadas na produção e consumo de sinais. Portanto, não há separação entre "realidade" e representação simbólica, quando os críticos da mídia eletrônica argumentam que o novo ambiente simbólico não representa a "realidade", eles implicitamente referem-se a uma absurda ideia primitiva da experiência real "não-codificada" que nunca existiu. Todas as realidades são comunicadas por intermédio de símbolos. E na comunicação interativa humana, independentemente do meio, todos os símbolos são, de certa forma, deslocados em relação ao sentido semântico que lhes são atribuídos. De certo modo, toda a realidade é percebida de maneira virtual. O que caracteriza o novo sistema de comunicação, baseado na integração em rede digitalizada de múltiplos modos de comunicação, é sua capacidade de inclusão e abrangência de todas as expressões culturais (SAVAZONE R e COHN S, 2009).

Como uma tendência histórica, as funções e processos dominantes na era da tecnologia estão cada vez mais organizados em redes. As redes constituem a nova morfologia social de nossas sociedades e a difusão da lógica de rede modifica substancialmente a operação e os resultados dos processos produtivos e da experiência, do poder e da cultura (SAVAZONE R e COHN S, 2009).

A inclusão/exclusão em redes e a arquitetura das relações entre elas, possibilitadas por tecnologias da informação que operavam à velocidade da luz, configuram os processos e funções predominantes em nossas sociedades. Redes são estruturas abertas capazes de expandir de forma ilimitada, integrando novos nós desde que consigam comunicar-se dentro da rede, ou seja, desde que compartilhem os mesmos códigos de comunicação. No âmbito cultural, isso resulta em expansão, reconhecimento, compartilhamento de pensamentos, de ações e de instituições. Essas potencialidades únicas que as redes sociais juntamente com a tecnologia trazem, fortalecem e somam cada vez mais aos processos e manifestações culturais (LÉVY, 1999).

\section{CONSIDERAÇÕES FINAIS}

A democratização das tecnologias da informação proporciona avanços importantes para a humanidade, resultando em ferramentas que são utilizadas para a promoção de uma comunicação mais abrangente. A tecnologia, em especial as redes sociais vem sendo apropriado por diferentes setores, e incorpora perspectivas diversas sobre o impacto das tecnologias digitais e da conexão em rede na sociedade, trazendo à tona mudanças concretas, reais e muito práticas em relação a tudo que está acontecendo no mundo, prova disto é o crescimento em massa das suas redes. Por esta razão, esses espaços se tornam cada vez mais fortes e presentes no cotidiano das pessoas, transformando-se em uma comunidade forte e rica em potencialidades, que podem ser utilizadas por diversos setores, seja para o desenvolvimento regional ou para o fortalecimento de movimentos culturais. Os ambientes virtuais são espaços ricos em participação e engajamento popular, sendo um local promissor para se promover a cultura. As redes sociais proporcionam a empresas e Instituições, possibilidades de organização e promoção desses órgãos. A tecnologia aliada a comunicação digital se torna cada vez mais uma ferramenta decisiva para o sucesso de empresas, pessoas

REAS/EJCH | Vol. Sup. 22 | e587| DOI: https://doi.org/10.25248/reas.e587.2019 Página $\mathbf{5}$ de $\mathbf{6}$ 
públicas e organizações sem fins lucrativos. Por se tratar de um espaço participativo, democrático e barato, para o setor cultural, traz inúmeros benefícios quando utilizado de forma correta. Os principais propósitos desta pesquisa foram de apontar as redes sociais e midiáticas que podem contribuir para maior visibilidade a Projetos Culturais. Para tal, sugerimos o uso da rede social Facebook para a promoção e difusão das ações de entidades filantrópicas.

\section{REFERÊNCIAS}

1. AGUIAR S. Redes Sociais na internet. Desafios à pesquisa. In: XXX CONGRESSO BRASILEIRO DE CIÊNCIAS DA COMUNICAÇÃO. 2007. Anais... Santos: Intercom, 2007ㄹ. Disponível em: http://www.sitedaescola.com/downloads/portal_aluno/Maio/Redes\%20sociais\%20na\%20internet\%20desafios\%20\%E0\%20pesquisa.pdf>. Acessado em: 26 de Junho 2018.

2. BURKE P. Uma história social da mídia: de Gutenberg à Internet. Tradução: DIAS, Maria Carmelita Pádua. Revisão técnica: VAZ, Paulo. 2a. Edição. Rio de Janeiro: Jorge Zahar Editor, 2006.

3. COSTA AC. Redes Sociais. Editora Nova terra, Rio de janeiro RJ, 2015.

4. CHAUÍ M. Conformismo e resistência: aspectos da cultura popular no Brasil. São Paulo: Brasiliense, 1986.

5. EMPRESA ComScore. Dados sobre usuário das redes sociais no Brasil Disponível em: <http://www.comscore.com/por/Imprensa-e-eventos/Press-Releases/2014/5/Estudo-da-comScore-Brazil-DigitalFuture-in-Focus-2014-esta-disponivel>. Acessado em: 25 de Julho de 2018.

6. GABRIEL M. Marketing na Era Digital: Conceitos, plataformas e estratégias. Editora Novatec, São Paulo, 2010.

7. LEVY P. Cibercultura. Trad. Carlos Irineu da Costa. $2^{\circ}$ Edição. São Paulo: Editora 34, 2000.

8. LEVY P. Inteligência coletiva. Editora: Edições Loyola, 2007.

9. KOTLER K, SETEAWAN P, HERMAWAN I. Marketing 4.0. GMT Editores Ltda. Rio de Janeiro, 2017.

10. OLIVEIRA IL, MARCHIORI M. Redes sociais, comunicação, organizações. Editora 34. 1999.

11. SAVAZONE R, COHN S. Cultura digital. Disponível em <https://pt.scribd.com/doc/78742907/CulturaDigital-br > Acessado: em 17 jul. 2018. 